\title{
Caracterización de las conductas suicidas en adolescentes de la zona de influencia del volcán Galeras, Nariño, Colombia*11
}

\author{
Fredy Hernán Villalobos-Galvis \\ Doctor en Psicología Clínica y de la Salud \\ Universidad de Nariño \\ Colombia \\ Correo electrónico: fhvillalobos@udenar.edu.co
}

Elizabeth Ojeda-Rosero

Doctora en Psicología

Universidad de Nariño

Colombia

Correo electrónico: deliza75@yahoo.com

\section{Edwin Gerardo Luna-Tascón}

Magister en Educación desde la Diversidad Universidad de Nariño

Colombia

Correo electrónico: lunaedwingerardo@gmail.com

Recibido: 22/08/2018

Evaluado: 04/12/2018

Aceptado: 15/01/2019

\section{Resumen}

Las conductas suicidas se presentan de manera especial en la adolescencia y cuando las personas enfrentan circunstancias altamente demandantes. El objetivo de este trabajo fue caracterizar la prevalencia de la conducta suicida en adolescentes de la Zona de Amenaza Alta del Volcán Galeras - ZAVA, y establecer su asociación con variables demográficas y psicológicas. La muestra estuvo conformada por 154 adolescentes matriculados en instituciones educativas ubicadas en la ZAVA, en quienes se evaluó la presencia de conductas suicidas, depresión, desesperanza, autoestima, apoyo social y eventos vitales. La prevalencia en el transcurso de la vida para ideas suicidas fue de $17.5 \%$, para planes $6 \%$ y para intentos $8 \%$. Las conductas suicidas se relacionaron con sexo, depresión, desesperanza y número de eventos vitales estresantes. Se concluye que los adolescentes constituyen una población con riesgo de conductas autolíticas, demandando acciones que permitan enfrentar esta situación, desde una mirada pública de la salud mental.

\section{Palabras clave}

Suicidio, salud mental, adolescentes, experiencias de vida, riesgo volcánico, reasentamiento por desastre.

11 Para citar este artículo: Villalobos-Galvis, F.H., Ojeda-Rosero, E., \& Luna-Tascón, E.G. (2019). Caracterización de las conductas suicidas en adolescentes de la zona de influencia del volcán Galeras, Nariño, Colombia. Informes Psicológicos, 19(2), pp. 163-180 http://dx.doi.org/10.18566/infpsic.v19n2a011

Nota: Este trabajo es parte del informe final del proyecto "Estudio epidemiológico de la conducta suicida en adolescentes escolarizados de la zona de amenaza volcánica alta del volcán Galeras", financiado por el Sistema de Investigaciones de la Universidad de Nariño. 


\title{
Characterization of suicidal behaviors in adolescents from the area of influence of the Galeras volcano, Nariño, Colombia
}

\begin{abstract}
Suicidal behaviors occur mainly during adolescence and when people face highly demanding circumstances. The objective of this work was to characterize the prevalence of suicidal behavior in adolescents of the High Threat Zone of the Galeras Volcano -ZAVA-, and to establish its association with demographic and psychological variables. The sample consisted of 154 adolescents enrolled in educational institutions located in the ZAVA, in whom the presence of suicidal behavior, depression, despair, self-esteem, social support and vital events were evaluated. The prevalence for suicidal ideas in the course of life was $17.5 \%$, for plans $6 \%$ and for attempts $8 \%$. Suicidal behaviors were related to sex, depression, hopelessness and number of stressful life events. It is concluded that adolescents constitute a population with a risk of autolytic behaviors, demanding actions to face this situation, from a public view of mental health.
\end{abstract}

Keywords suicide, mental health, adolescents, life experiences, volcanic risk, resettlement by disaster

\section{Caracterização das condutas suicidas em adolescentes na zona de influência do Vulcão Galeras, Narinho, Colômbia \\ Resumo}

As condutas suicidas apresentam-se de maneira especial na adolescência e quando as pessoas afrontam circunstâncias demandantes. 0 objetivo deste trabalho foi caracterizar a prevalência da conduta suicida em adolescentes da zona de alto risco do vulcão Galeras -ZAVA, e estabelecer sua associação com variáveis demográficas e psicológicas. A amostra esteve conformada por 154 adolescentes cadastrados em instituições educativas localizadas na zona de alto risco (ZAVA), nos quais avaliou-se a presencia de condutas suicidas, depressão, desesperança, autoestima, suporte social e eventos vitais. A prevalência no percurso da vida para ideias suicidas foi de $17.5 \%$, para planos foi um $6 \%$ e para tentativas foi de um $8 \%$. As condutas suicidas foram relacionadas com sexo, depressão, desesperança e um número de eventos vitais estressantes. Conclui-se que os adolescentes constituem uma população com risco de condutas autolíticas, demandando ações que permitam afrontar esta situação desde uma perspectiva de saúde mental pública.

Palavras chave suicídio, saúde mental, adolescentes, experiencias de vida, risco vulcânico, reassentamento por desastre.. 


\section{ntroducción}

La Zona de Amenaza Alta del Volcán Galeras (ZAVA), ubicada en el departamento de Nariño-Colombia, es el área más cercana al cráter activo, en la que pueden ocurrir fenómenos volcánicos como: flujos de lava, piroclásticos o de lodo, proyectiles balísticos (caídas de piedras), onda de choque y alta concentración de gases tóxicos. En esta zona se encuentran algunos sectores específicos de los municipios de Pasto, Nariño, La Florida, Sandoná y Consacá (Consejo Nacional de Política Económica y Social -CONPES-, 2007).

Considerando que la actividad volcánica registró un incremento durante el mes de noviembre de 2005, el Gobierno Nacional declaró situación de desastre de carácter departamental para los municipios de Pasto, Nariño y La Florida (República de Colombia, 2005) y luego definió los instrumentos necesarios para implementar un Plan de Reasentamiento para los habitantes de la ZAVA del Volcán Galeras (República de Colombia, 2008). Sin embargo, los procesos de movilización social y de resistencia civil de las comunidades que habitaban la ZAVA, entre otros aspectos, llevó a que la implementación del denominado "Plan Galeras" se diera de manera parcial y demorada, hasta que la ley 1523 derogó la declaratoria de desastre (Congreso de Colombia, 2012).

Las consecuencias sociales, políticas y económicas de la declaratoria inicial de desastre y del inminente plan de reasentamiento para los habitantes de la ZAVA fueron variadas, incluyendo la salud mental de los habitantes (Ojeda, 2008). El reporte de las manifestaciones emocionales y conductuales que sucedieron después de la declaratoria de desastre y el subsecuente temor a la puesta en marcha del plan de reasentamiento, permitían pensar que la salud mental de los adolescentes habitantes de la ZAVA podía haberse afectado, y con ello el incremento del riesgo de conductas autolíticas, razón por la cual el presente trabajo buscó establecer la prevalencia de conductas suicidas en este grupo poblacional.

La conducta suicida, en tanto es un fenómeno multidimensional, al ser el producto de una interacción compleja de determinantes ambientales, orgánicos, psicológicos y sociológicos (OMS, 2000), requiere de un abordaje complejo, en el que se hace necesario el trabajo de identificación de los factores de riesgo y protección para cada grupo poblacional (Cortés, Aguilar, Suárez, Rodríguez \& Durán, 2011).

La conducta suicida abarca, entre otras, la ideación, el plan, el intento y el suicidio consumado (Villalobos, 2008). La Organización Mundial de la Salud (OMS, 2018) manifiesta que cada año aproximadamente 800.000 personas se suicidan, la frecuencia del intento es 20 veces mayor en comparación con la del suicidio y para las personas entre 15 y 29 años se convierte en la segunda causa de defunción. Según el Instituto Colombiano de Medicina Legal y Ciencias Forenses (Montoya, 2018), en Colombia se registraron 2571 defunciones por suicidio en el año 2017, lo que representa una tasa de 5,72/100.000 habitantes, manteniendo la tendencia ascendente vista desde el año 2013. En 2017 las personas entre 20 y 24 años alcanzaron la tasa más 
alta, seguidas del grupo poblacional de 18 a 19 años, mientras que los hombres presentaron mayores casos de suicidio (Montoya, 2018). En Nariño, en el año 2016 se reportaron 129 suicidios, el mayor número se evidenció en adolescentes entre los 15 y 19 años de edad, seguido de adultos jóvenes entre los 20 y 24 años; los hombres presentaron mayores casos de suicidio (DANE, 2016).

Con respecto a los intentos de suicidio, en Colombia entre los años 2009 y 2015 se presentaron 10.325 casos de lesiones autoinfligidas, fenómeno que aumentó entre 2010 y 2016; las personas de 15 a 19 años y las mujeres presentaron mayores tasas de intento (Ministerio de Salud y Protección Social, 2017). En Nariño, se identificó que: los casos reportados con más frecuencia estaban entre los 13 y los 16 años, las mujeres eran el grupo más prevalente, los métodos más utilizados eran la ingestión de tóxicos y el ahorcamiento; y las causas más recurrentes eran el ambiente familiar (padres separados o divorciados), conflictos de pareja y maltrato (abuso sexual, físico y verbal) (Jiménez, Hidalgo, Camargo \& Dulce, 2014).

Finalmente, la Encuesta Nacional de Salud Mental (Ministerio de Salud, 2015) identificó que la ideación suicida es el evento más frecuente con un $6.6 \%$, seguido del intento con un $2.6 \%$ y $1.8 \%$ para el plan suicida. Específicamente en los adolescentes, el comportamiento suicida más prevalente fue la ideación (Ministerio de Salud, 2015).

De acuerdo con las cifras anteriores, los adolescentes se convierten en uno de los segmentos poblacionales con mayor riesgo de presentar conductas suicidas; específicamente, en esta etapa evolutiva se presenta un incremento de estresores o responsabilidades individuales que, sumadas a la poca experiencia y la falta de madurez, generan momentos de angustia, soledad y frustración, desconocidas y difíciles de manejar (Cortés, 2013). De igual manera, factores como el consumo de sustancias psicoactivas, alteraciones de la conducta y de las emociones, pérdida reciente de una persona amada, autoimagen disminuida, tentativas previas de suicidio, historia de abuso físico o sexual, y exposición a eventos vitales adversos, entre otros, aumentan las probabilidades de presentar una conducta suicida (Pérez, Téllez, Vélez \& Ibáñez, 2012).

Según lo anterior, el presente trabajo buscó determinar la prevalencia de los comportamientos suicidas en adolescentes escolarizados que habitan en la ZAVA del Volcán Galeras, identificar las variables demográficas y psicológicas asociadas con las conductas suicidas y caracterizar los intentos de suicidio que se pudieran presentar en la población estudiada.

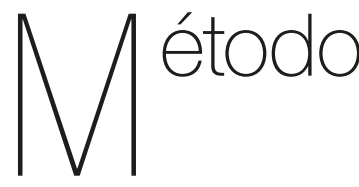

\section{Tipo de estudio}

Este trabajo es un estudio descriptivo, pues buscó caracterizar la conducta suicida de los estudiantes que vivían en municipios o corregimientos de la ZAVA del Volcán Galeras. Además, es correlacional al buscar establecer posibles relaciones entre la conducta suicida y algunas variables psicológicas y demográficas de los 
participantes. Finalmente, es un estudio transversal (Gómez \& Villalobos, 2014), en tanto los datos fueron recolectados en el mes de noviembre del año 2011, en una sola ocasión.

\section{Instrumentos}

Se hizo uso de una encuesta sobre datos sociodemográficos y se aplicaron los siguientes instrumentos de medida:

\section{Cédula de Indicadores Parasuicidas (CP):}

Está integrada por preguntas sobre lesiones autoinfligidas deliberadamente e intentos suicidas durante la vida (González et al., 2005). La CIP ha sido validada en estudios previos (Unikel, Gómez \& González-Forteza, 2006).

\section{Escala de Depresión del Centro de Estudios Epidemiológico (CES-D):}

Mide síntomas de depresión en población general, durante las últimas dos semanas (Radloff, 1977) y presenta buenos indicadores de fiabilidad y validez en adolescentes y jóvenes (Veytia, Fajardo, Guadarrama \& Escutia, 2016; Villalobos \& Ortiz, 2012).

\section{Escala de Desesperanza de Beck} (Beck Hopelessness Scale - BHS):

Evalúa los tres aspectos clave en la desesperanza: expectativas, sentimientos acerca del futuro y pérdida de la motivación; posee una validez factorial satisfactoria y alto grado de consistencia interna (González, 2009).
Escala de Autoestima de Rosenberg (AER):

Contiene 10 reactivos que abordan tanto la aceptación, como los sentimientos de respeto de sí mismo; ha demostrado adecuados niveles de fiabilidad y validez en contextos diferentes (Cogollo, Campo \& Herazo, 2015).

\section{Escala de Apoyo Social (AS):}

Se usó una versión de 13 ítems que versan sobre la percepción de personas disponibles para brindar apoyo emocional, instrumental e informacional, en las áreas personal, laboral y familiar (Matud, 1998). Es una escala unifactorial que, en diferentes contextos, ha evidenciado adecuada fiabilidad y validez (Matud et al., 2002).

\section{Cuestionario de Eventos Vitales Estresantes para Adolescentes de la Universidad de Nariño - CEVE-UDENAR:}

Identifica y evalúa el impacto de 28 eventos vitales, frente a los que el examinado debe responder si los ha vivido o no en los últimos 6 meses y, en caso positivo, especificar la magnitud de la afectación producida. Ha demostrado buenas propiedades psicométricas en población adolescente (Delgado \& Santacruz, 2012).

\section{Encuesta de evaluación de los efectos psicosociales del posible reasentamiento familiar}

Instrumento elaborado para la presente investigación, consta de 17 reactivos que evalúan la percepción de 
los estudiantes sobre el reasentamiento familiar, los cambios que se pueden presentar en su vida a partir del reasentamiento, el grado de preparación personal, familiar y comunitario ante el reasentamiento; y, finalmente, la actitud familiar frente al posible reasentamiento. La construcción del instrumento implicó la definición del atributo, la elaboración de los ítems y la revisión por integrantes del equipo investigador. Se obtuvo una confiabilidad moderada (Alpha de Cronbach $=.78$ ), según los mismos datos del presente estudio.

\section{Participantes}

Participaron 154 estudiantes de tres instituciones educativas situadas en los municipios de La Florida y Nariño y en el corregimiento de Mapachico (municipio de Pasto), ubicadas en la ZAVA del Volcán Galeras, elegidos aleatoriamente del listado de estudiantes por curso. El 57.1\% eran mujeres y las edades se encontraban entre los 11 y los 19 años ( $M=14.8$, DS=1.79), siendo más frecuentes las de 13 y 15 años. Los estudiantes estaban matriculados en los grados $6^{\circ}$ a $11^{\circ}$ de secundaria.

\section{Técnicas de análisis}

Las variables de salud mental fueron categorizadas por niveles. Los análisis estadísticos se elaboraron con el programa IBM@ SPSS $®$ Statistics v22 (IBM, 2013), con el cual se obtuvieron porcentajes y frecuencias absolutas de las variables objeto de estudio, además de las tablas de contingencia sobre las cuales se calculó el coeficiente Chi Cuadrado de Pearson $\left(\chi^{2}\right)$. De igual manera, por medio del software G*Power v3.1.9.2 (Faul, Erdfelder, Lang \& Buchner, 2007) se calculó la potencia estadística (PE) post-hoc de cada $\chi^{2}$, entendida ésta como el grado de validez de los resultados, dados el tamaño de muestra, el error $\alpha$ y el tamaño del efecto (w) (Cárdenas \& Arancibia, 2014). En los resultados sólo se presentan los cruces que cumplieron con los siguientes criterios: $\alpha<.05, w>.3$ y PE $>$.8.

\section{Aspectos éticos}

El presente estudio fue aprobado por el Comité de Ética en Investigación de la Universidad de Nariño, siendo catalogado como un estudio de riesgo mínimo (Ministerio de Salud de Colombia, 1993). Posteriormente, se obtuvo la aprobación de las directivas de las tres instituciones educativas, quienes facilitaron el acceso a los docentes, padres de familia/acudientes y estudiantes. Se hizo un muestreo aleatorio simple por cada institución y a los estudiantes elegidos se les invitó a participar, para lo cual se debía contar con el asentimiento informado, si los estudiantes eran menores de edad, y con el consentimiento informado para sus padres/acudientes o estudiantes mayores de edad. Los resultados preliminares de este trabajo se presentaron a las instituciones educativas que participaron de la investigación, además se llevó a cabo un taller con representantes del sector salud y de las instituciones educativas, con el propósito de favorecer la generación de acciones pertinentes frente al problema de las conductas suicidas. 
Resultados

\section{Prevalencia de las conductas suicidas durante la vida}

Al indagar por la presencia de conductas suicidas a lo largo de la vida en los estudiantes, se encontró mayor frecuencia de ideas, seguidas de intentos y de planes, identificando una prevalencia de conductas suicidas durante la vida de 31.2\% (ver Tabla 1).

Tabla 1.

Tabla de contingencia entre sexo y conducta suicida

\begin{tabular}{cccccc}
\hline \multicolumn{5}{c}{ Conducta Suicida } \\
\hline Sexo & Ninguna & Ideas & Planes & Intentos & N \\
\hline Mujer & $59.1 \%$ & $20.5 \%$ & $9.1 \%$ & $11.4 \%$ & 88 \\
Hombre & $81.8 \%$ & $13.6 \%$ & $3.0 \%$ & $1.5 \%$ & 66 \\
\hline Total & $68.8 \%$ & $17.5 \%$ & $6.5 \%$ & $7.1 \%$ & 154 \\
\hline
\end{tabular}

Fuente: Elaboración propia

\section{Factores sociodemográficos y conductas suicidas}

Se encontró que las mujeres tienden a presentar una mayor proporción de todas las conductas suicidas (ver Tabla 1), asociación que resultó estadísticamente significativa $\left(\chi^{2}=11.085 ; \mathrm{gl}=3 ; \mathrm{p}<.011\right.$, $\mathrm{W}=.38$; $\mathrm{PE}=.985)$. Así, las mujeres reportaron 1.5 veces más ideas, 3 veces más planes y 7.6 veces más intentos que los hombres.

La ideación suicida es reportada de manera más frecuente por quienes cursan grado décimo, con una evidente tendencia ascendente en los años anteriores. Los planes suicidas tienen el punto de mayor incidencia en los grados $7^{\circ} \mathrm{y}$ $9^{\circ}$. Finalmente, el mayor número de intentos de suicidio es reportado por los alumnos de grado $8^{\circ}$, aunque los intentos se reportan desde el grado $6^{\circ}$ (ver Figura 1).

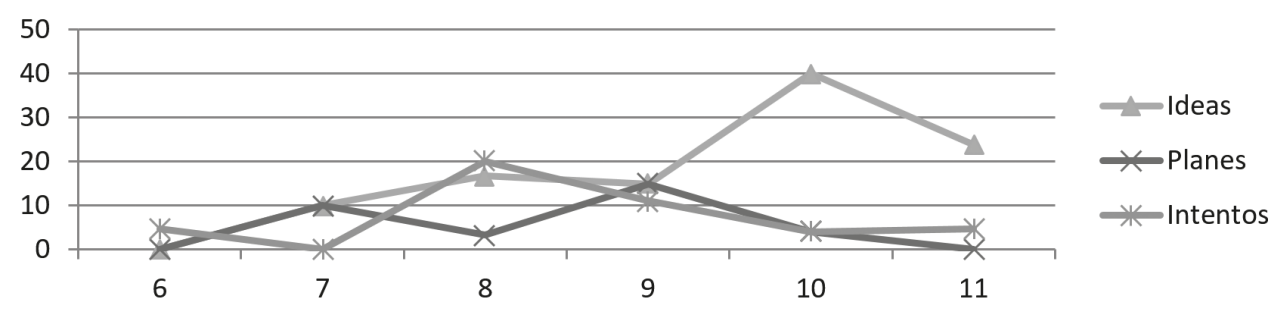

Figura 1. Porcentaje de conductas suicidas reportadas, según grado escolar.

Se encontró que los adolescentes que conocieron a alguien que tuvo conductas suicidas, a su vez presentaron mayores proporciones de conductas suicidas (ver Tabla 2), especialmente de planes e ideas $\left(\chi^{2}=13.169 ; g l=3 ; p<.004 ; w=.39 ; P E\right.$ 
$=.989$ ). Adicionalmente, el $52.6 \%$ de los estudiantes conocían a personas que se suicidaron o que lo intentaron. Las personas reportadas fueron el padre, la madre o los hermanos en un 2.1\%; otros familiares en un 6.2\%, amigos o novios en el $14.3 \%$ y conocidos en el $32.5 \%$.

Tabla 2.

Tabla de contingencia entre conocer a un suicida y la presencia de conducta suicida

\begin{tabular}{crrrrr}
\hline \multicolumn{5}{c}{ Conducta Suicida } \\
\hline $\begin{array}{c}\text { ¿Conoció a } \\
\begin{array}{c}\text { alguien con } \\
\text { conductas } \\
\text { suicidas? }\end{array}\end{array}$ & Ninguna & Ideas & Planes & Intento & N \\
\hline No & $82.2 \%$ & $11 \%$ & $1.4 \%$ & $5.5 \%$ & 73 \\
Sí & $56.8 \%$ & $23.5 \%$ & $11.1 \%$ & $8.6 \%$ & 81 \\
\hline Total & $68.8 \%$ & $17.5 \%$ & $6.5 \%$ & $7.1 \%$ & 154 \\
\hline
\end{tabular}

Fuente: Elaboración propia

\section{Conductas suicidas y efectos psicosociales hacia el posible reasentamiento familiar}

Al indagar sobre los posibles cambios negativos que se darían en sus vidas a partir del posible reasentamiento, un 35\% de los estudiantes consideró que se afectaría su vida personal, un $48 \%$ su vida social, un $46.8 \%$ su vida sentimental, un $41 \%$ su vida familiar, un $48 \%$ las condiciones económicas familiares y un $45 \%$ la vida de la comunidad. En cuanto al grado de preparación que se tenía frente a un posible reasentamiento, se encontró que sólo un $17.5 \%$ de los participantes decía no estar preparado personalmente, un $22 \%$ consideraba que la familia no estaba preparada, un $42 \%$ afirmaba que no estaba preparada la comunidad y un $20 \%$ decía que no estaban preparadas las entidades estatales.

El posible reasentamiento generaba principalmente reacciones de tristeza (74.7\%), miedo (51.9\%), ira (23.4\%) y alegría (17\%). Igualmente, se encontró que el $48 \%$ de las familias estaban en contra del reasentamiento (seguido por un $41.6 \%$ cuya respuesta fue "no sé") y que el $42.2 \%$ de las familias estaban unidas en cuanto a su decisión específica frente al reasentamiento. No obstante lo anterior, ninguna de las variables consideradas como efectos psicosociales del posible reasentamiento familiar demostró una relación estadísticamente significativa con las conductas suicidas.

\section{Indicadores de salud mental}

Se estableció que los mayores niveles de riesgo en salud mental estaban asociados con depresión, seguida de eventos vitales estresantes y desesperanza. En cuanto a las variables protectoras, un 42.8\% poseía niveles adecuados de autoestima y un $63.6 \%$ buenos niveles de apoyo social (ver Tabla 3).

Tabla 3.

Niveles de variables de riesgo y protectoras

\begin{tabular}{cccccc}
\hline Nivel & $\begin{array}{r}\text { Muy } \\
\text { Bajo }\end{array}$ & Bajo & Medio & Alto & $\begin{array}{c}\text { Muy } \\
\text { Alto }\end{array}$ \\
\hline Desesperanza & 44.8 & 25.3 & 14.3 & 8.4 & 7.1 \\
$\begin{array}{c}\text { Depresión } \\
\begin{array}{c}\text { Eventos vitales } \\
\text { estresantes }\end{array}\end{array}$ & 31.7 & 16.4 & 23.7 & 23 & 15.1 \\
$\begin{array}{c}\text { Autoestima } \\
\text { Apoyo social }\end{array}$ & 17.7 & 19.5 & 19.1 & 17.1 & 14.5 \\
\hline
\end{tabular}

Fuente: Elaboración propia 
Al analizar los datos de las variables de riesgo y la presencia de conductas suicidas, se encontró una relación significativa $\left(\chi^{2}=16.65 ; \mathrm{gl}=6 ; \mathrm{p}=.011, \mathrm{w}=.37 ; \mathrm{PE}=\right.$ .982) entre los niveles de desesperanza y los tipos de conductas suicidas reportadas por los estudiantes. Concretamente, el $20.8 \%$ de quienes tenían alto nivel de desesperanza, presentaron intentos de suicidio, mientras que el $73.1 \%$ de quienes tenían niveles bajos de desesperanza no presentó ninguna conducta suicida (ver Tabla 4).

Tabla 4.

Relación entre niveles de desesperanza y conductas suicidas

\begin{tabular}{cccccc}
\hline Desesperanza & Ninguna & Ideas & Planes & Intentos & N \\
\hline Bajo & $73.1 \%$ & $19.4 \%$ & $3.7 \%$ & $3.7 \%$ & 108 \\
Medio & $54.5 \%$ & $22.7 \%$ & $13.6 \%$ & $9.1 \%$ & 22 \\
Alto & $62.5 \%$ & $4.2 \%$ & $12.5 \%$ & $20.8 \%$ & 24 \\
\hline
\end{tabular}

Fuente: Elaboración propia

En lo referente a la relación entre depresión y conductas suicidas, se halló que el $91.4 \%$ de quienes tenían bajos niveles de depresión no presentó ninguna conducta autolítica, mientras que el 25\% de quienes presentaron niveles medios tuvieron ideas suicidas; por su parte, el $15.5 \%$ y el $13.8 \%$ de quienes tuvieron altos niveles de depresión, reportaron planes e intentos de suicidio, respectivamente $\left(\chi^{2}=31.48 ; \mathrm{gl}=6 ; \mathrm{p}=.000, \mathrm{w}=.46 ; \mathrm{PE}\right.$ $=.999)$ (ver Tabla 5).

Tabla 5.

Relación entre niveles de depresión y conductas suicidas

\begin{tabular}{cccccc}
\hline Depresión & Ninguna & Ideas & Planes & Intentos & N \\
\hline Bajo & $91.4 \%$ & $8.6 \%$ & $.0 \%$ & $.0 \%$ & 58 \\
Medio & $66.7 \%$ & $25.0 \%$ & $2.8 \%$ & $5.6 \%$ & 36 \\
Alto & $50.0 \%$ & $20.7 \%$ & $15.5 \%$ & $13.8 \%$ & 58 \\
\hline
\end{tabular}

Fuente: Elaboración propia
La cantidad de eventos vitales estresantes experimentados en los últimos 6 meses fue otra variable relevante, al hallarse una relación significativa $\left(\chi^{2}=14.34\right.$; $g l=6 ; p=.026, w=.3 ; P E=.91)$ con las conductas suicidas. Se encontró que la mayor presencia de intentos de suicidio se dio en estudiantes con nivel medio de Eventos Vitales Estresantes (EVE), mientras que en los niveles altos se ubicaron las mayores prevalencias de ideas y planes suicidas (ver Tabla 6).

Tabla 6.

Prevalencias de ideas y planes suicidas

\begin{tabular}{cccccc}
\hline Nivel EVE & Ninguna & Ideas & Planes & Intentos & N \\
\hline Bajo & $78.7 \%$ & $16.0 \%$ & $.0 \%$ & $5.3 \%$ & 75 \\
Medio & $65.5 \%$ & $13.8 \%$ & $10.3 \%$ & $10.3 \%$ & 29 \\
Alto & $56.3 \%$ & $22.9 \%$ & $14.6 \%$ & $6.3 \%$ & 48 \\
\hline
\end{tabular}

Fuente: Elaboración propia

Finalmente, ninguna de las variables protectoras (apoyo social y autoestima) mostró relación significativa $(p<$.05) con la prevalencia de conductas suicidas en esta muestra.

\section{Eventos vitales estresantes y Conducta suicida}

De los EVE evaluados, cuatro mostraron asociaciones estadísticamente significativas $(p<.05)$ con la presencia de conductas suicidas. Con respecto a los intentos de suicidio, el principal EVE fue la presencia de conductas suicidas (intentos o suicidios) en familiares cercanos. En cuanto a planes suicidas, aparecieron como relevantes: problemas con la ley, el cambio de ciudad, barrio o casa; y las 
peleas con un ser querido. Por último, en relación con las ideas suicidas, aparecieron como relevantes las conductas suicidas en familiares y las peleas con seres queridos (ver Tabla 7).

Tabla 7.

Principales Eventos Vitales Estresantes asociados a las conductas suicidas

\begin{tabular}{|c|c|c|c|c|c|c|c|c|}
\hline \multirow[b]{2}{*}{ Eventos } & \multicolumn{4}{|c|}{ Conducta Suicida } & \multicolumn{4}{|c|}{ Estadísticos } \\
\hline & Ninguna & Ideas & Plan & Intento & $\mathrm{n}$ & $\mathrm{Chi}^{2}$ & w & $\mathrm{PE}$ \\
\hline Conductas suicidas en familiares & $37.5 \%$ & $29.2 \%$ & $12.5 \%$ & $20.8 \%$ & 24 & $15.29^{* *}$ & .32 & .923 \\
\hline $\begin{array}{l}\text { Peleas o discusiones graves } \\
\text { con ser querido }\end{array}$ & $54.9 \%$ & $24.4 \%$ & $11 \%$ & $9.8 \%$ & 82 & $17.96^{\star \star}$ & .44 & .998 \\
\hline Problemas con la ley & $64.3 \%$ & $7.1 \%$ & $28.6 \%$ & $0 \%$ & 14 & $13.65^{\star *}$ & .30 & .896 \\
\hline Cambio de casa, barrio o ciudad & $62.5 \%$ & $16.7 \%$ & $16.7 \%$ & $4.2 \%$ & 48 & $12.41^{\star *}$ & .33 & .943 \\
\hline
\end{tabular}

\section{Características de los intentos de suicidio}

Entre los casos que reportaron haber tenido algún intento de suicidio durante su vida $(n=11)$, se encontró que la cantidad de intentos estuvo entre uno y 10, con un promedio de 4.67 por persona (DS = 3.57). Igualmente, se encontró que el $50 \%$ de los casos tuvieron su último intento hace más de un año, el 17\% hace más de seis meses y el $8.3 \%$ en los últimos seis meses. Sin embargo, resulta preocupante que el 25\% de los casos de intento se habían presentado en el último mes.

De otro lado, se evidenció que la edad promedio del primer o único intento fue de 13.58 años (DE = 2.27), oscilando entre los 10 y los 17. La edad crítica fue de 13 años, en la cual se ubicó el 33.3\% de los casos. Solamente en el $16.7 \%$ de los casos había una intención clara de morir, mientras que a un 33.3\% no le importaba si vivía o moría y el 50\% restante no quería morir. El método más utilizado fue el corte con objetos cortopunzantes (83.3\%) y en segundo lugar la ingesta de pastillas o medicamentos (33\%).

Con respecto a la finalidad del intento, se halló que el mayor porcentaje quería dejar de ser una carga para sus seres queridos (33\%), así como terminar con los problemas que se vivían en ese momento (33\%), lo cual se relaciona con quitarse un sufrimiento (25\%), seguido de autocastigarse (25\%). Finalmente, llama la atención que se reporta el "placer/gusto" como otra finalidad del intento de suicidio. Adicionalmente, los estudiantes reportaron que en el $83.3 \%$ de las ocasiones nadie más se enteró de lo sucedido, mientras que cuando lo comentaron lo hicieron principalmente con los amigos o con un guía espiritual (25\% cada uno) y en menor medida solicitaron ayuda profesional $(16.7 \%)$ 


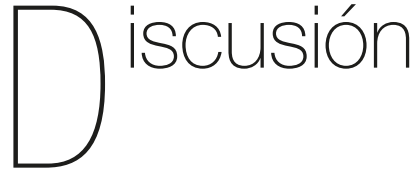

El propósito central del presente estudio fue caracterizar la prevalencia durante la vida de las conductas suicidas en estudiantes de instituciones educativas ubicadas en la ZAVA del Volcán Galeras (Nariño-Colombia) y establecer sus posibles relaciones con diferentes variables demográficas y psicológicas.

En primer lugar, se encontró que la prevalencia de las conductas suicidas (ideas $17.5 \%$, planes $6 \%$ e intentos $8 \%$ ) es muy similar a la encontrada en otros estudios realizados en Nariño (Villalobos, 2009; Chamorro \& Cristancho, 2010), CoIombia (Toro, Paniagua, González \& Montoya, 2009; Pérez, Martínez, Vianchá \& Avendaño, 2017) y Latinoamérica (PérezChan, 2015; Bazán, Olórtegui, Vargas \& Huayanay, 2016; Valdivia, Silva, Sanhueza, Cova \& Melipillán, 2015). Lo anterior permite afirmar que los estudiantes de las instituciones ubicadas en la ZAVA tienen un nivel de riesgo de suicidio similar al de estudiantes de otras ciudades y que las conductas suicidas tienen una presencia significativa en su vida, lo que lleva a considerarlos como un grupo de interés desde el ámbito de la salud pública, específicamente en cuanto a la prevención del suicidio.

Por otro lado, se encontró que las conductas suicidas siguen un patrón temporal, pues las ideas tienen un carácter ascendente a lo largo de la secundaria, los planes tienen su mayor incidencia en los grados séptimo y noveno, y los intentos llegan a su pico más alto en el grado octavo. Otros estudios han descrito las conductas suicidas según el grado de escolaridad, por ejemplo, Salas, Levette, Redondo y Luzardo (2017) y Chamorro y Cristancho (2010) establecieron que la ideación suicida prevalece en los grados octavo y noveno. De manera complementaria, la literatura en el tema demuestra que los grupos etarios de mayor riesgo varían de un contexto a otro, definiendo a la adolescencia como una etapa crítica para las diferentes conductas suicidas (Álvarez et al., 2017; Ceballos et al., 2015; Hernández, González \& López, 2013; Pérez et al., 2017; Piedrahita, García, Mesa \& Stivalis, 2011; Salas et al., 2017; Toro et al., 2009; Villalobos, 2009).

En conclusión, parecería que la adolescencia y la secundaria se convierten en etapas significativas en el desarrollo de las conductas suicidas. Al respecto, es importante la formulación de estudios longitudinales (e.g. cohortes) que permitan verificar esta posible tendencia evolutiva y, de encontrarse, formular programas de promoción y prevención con esa misma mirada de desarrollo (Sánchez, García \& Muela, 2014). Lo anterior, teniendo en cuenta que sería válido suponer que el riesgo de conductas suicidas cambia a lo largo de la vida de las personas y está relacionado con el tipo de estresores vitales que enfrentan, así como con sus recursos personales y de su medio social con los que cuentan en cada una de las etapas del desarrollo.

En cuanto a la asociación de variables demográficas y psicológicas con las conductas suicidas, inicialmente se encontró que el sexo es una variable que se relaciona con diferencias marcadas en el tipo de conducta presentada. Para este caso, las mujeres mostraron una mayor proporción 
de todas las conductas, reforzando la idea de que hay mayores prevalencias de conductas suicidas no letales en ellas, sobre todo en relación a la ideación y los intentos (Álvarez et al., 2017; Forero, Siabato \& Salamanca, 2017; Hernández et al., 2013; Pérez et al., 2012; Piedrahita et al., 2011; Salas et al., 2017; Valdivia et al., 2015; Vásquez, \& Quijano, 2013; Villalobos, 2009). Estos hallazgos reafirman la gran necesidad de abordajes con mirada de género, en los procesos de promoción de la salud mental, y específicamente, en los de prevención del suicidio (Sánchez et al., 2014).

Otra de las variables que se relaciona de manera relevante con las conductas suicidas es la imitación. Se encontró que quienes han conocido a otras personas que han realizado conductas suicidas, a su vez tienen una mayor tendencia a presentarlas; en este caso se observó una mayor proporción de planes e ideas. Estos resultados son congruentes con los encontrados por Sánchez, Guzmán y Cáceres (2005), en estudiantes universitarios; Piedrahíta, Paz y Romero (2012) en adolescentes escolarizados y Chamorro y Cristancho (2010) y Villalobos (2009) en adolescentes de Nariño. Lo anterior sugiere que el conocimiento de personas con conductas suicidas hace que dicho fenómeno sea más cercano al individuo, es decir, que se incremente la probabilidad de aparecer como una alternativa de afrontamiento en personas potencialmente suicidas. La imitación podría darse en términos de la evaluación que se hace de los estresores, de la evaluación de las capacidades personales para enfrentarlos o de las formas de afrontamiento (p.e. el suicidio) que se observan o se infieren del modelo suicida.
En cuanto a la asociación entre variables de riesgo y las conductas suicidas, en el presente estudio se encontró que el número de eventos estresantes está asociado al tipo de conducta suicida. Estudios previos han demostrado cómo algunos estresores son predictores relevantes de la conducta suicida, por ejemplo: la ruptura amorosa, el fallecimiento de un integrante de la familia o la mascota, la percepción de ir reprobando el año y el abuso sexual (Hernández et al., 2013; Martínez, 2016; Pérez et al., 2012; Pérez et al., 2017; Piedrahita, García, Mesa \& Rosero, 2011; Sánchez et al., 2014; Villalobos, 2009). Es amplia la evidencia que demuestra la relación entre eventos vitales estresantes y salud mental, específicamente en cuanto a las conductas suicidas (Siabato \& Salamanca, 2015; Vargas \& Saavedra, 2012; Vianchá, Bahamon \& Alarcón, 2013).

Adicionalmente, se encontró que mayores niveles de desesperanza se asociaban con mayor nivel de letalidad de las conductas suicidas, resultados coherentes con lo hallado por Córdova, Estrada y Velásquez (2013), quienes concluyen que existe mayor riesgo suicida cuando el adolescente presenta pensamientos y sentimientos de que no se puede modificar una situación. En este punto, existe una amplia y sólida evidencia del papel predictivo que tiene la desesperanza sobre las conductas suicidas en adolescentes (Forero et al., 2017; Jiménez et al., 2014; Pérez et al., 2012; Toro et al., 2009; Valdivia et al., 2015).

Por otro lado, la depresión es un correlato importante de las conductas suicidas. Se observó que a mayores niveles de depresión se encontraban mayores niveles de gravedad de la conducta suicida. 
En diferentes estudios se demuestra que esta variable se asocia de manera significativa con la intensidad o la gravedad de la conducta suicida, concluyendo que, a mayor nivel de depresión en los adolescentes, mayor riesgo suicida (Carvajal \& Caro, 2011; Miranda, Cubillas, Pérez \& Valdez, 2009; Villalobos \& Ortiz, 2012).

Por el contrario, resultó inesperada la falta de asociación entre el apoyo social y la autoestima (por un lado) y las conductas suicidas (por el otro). Efectivamente, son muchos los trabajos que han demostrado el papel protector que tienen estas variables frente a la conducta suicida (Ceballos et al., 2015; Martínez \& Robles, 2016; Miller, Esposito-Smythers \& Leictweis, 2015; Quinceno \& Vinaccia, 2013). No son claras las razones por las cuales no se encuentran los datos esperados, atribuyendo este resultado atípico al bajo tamaño de muestra obtenido, razón por la cual se recomienda la realización de nuevos estudios que confronten estos hallazgos.

En lo referente a los intentos de suicidio, se encontró que los principales métodos utilizados por los adolescentes de la ZAVA fueron el corte con objetos filudos y la ingesta de pastillas, situación que concuerda con lo hallado en otros estudios en lo referente a las heridas con objeto cortopunzante (Piedrahita et al., 2011) y la ingesta de medicamentos o envenamiento (Álvarez et al., 2017; Hernández et al., 2013; Martínez, 2016; Ministerio de la Salud y Protección Social, 2017; Vásquez, \& Quijano, 2013).

Adicionalmente, un aspecto llamativo es el rango de intentos de suicidio reportados (1-10), pues hace evidente el riesgo de que una vez presentado el intento, éste pueda repetirse una y otra vez, incrementando su riesgo de letalidad (Pérez et al., 2017; Pérez et al., 2012; Piedrahita et al., 2011; Vásquez, \& Quijano, 2013). Estos hallazgos validan la importancia de considerar a los estudiantes que han presentado esta conducta, como un grupo de alto riesgo para el suicidio.

En conclusión, se identifica que la ocurrencia y las características de las conductas suicidas en los adolescentes de la ZAVA, concuerdan con lo hallado en otras zonas del departamento de Nariño, de Colombia y de otros países. No obstante, esto no puede entenderse como una situación positiva, pues las conductas suicidas representan un alto riesgo para la salud pública, demandando en consecuencia, acciones orientadas a prevenir sus manifestaciones y a promover la generación de recursos personales, familiares y sociales que permitan a los adolescentes manejar adecuadamente sus estresores vitales, disminuyendo así la consideración de las conductas autolíticas como una estrategia efectiva para su manejo (Villalobos, 2008).

La principal limitación del presente estudio corresponde la alta tasa de no-respuesta hallada en la población (62\%). En este caso, la aplicación de los instrumentos se hizo al finalizar el año escolar, época en la que las condiciones culturales, institucionales y personales de los participantes impidieron la oportuna reposición del alto porcentaje de casos de no-respuesta. Este tipo de dificultades también ha sido reportada en otras publicaciones (Miranda et al., 2009), situación que afecta las inferencias estadísticas generadas. Ante esto, el uso de medidas de potencia estadística y tamaño de efecto post-hoc, permitieron tener un mayor grado de certeza 
sobre las afirmaciones enunciadas. Sin embargo, para futuros estudios se propone realizar la aplicación de instrumentos en una época que no afecte significativamente las dinámicas institucionales, unido a hacer mayores esfuerzos por facilitar la comprensión del estudio y motivar la participación de los educandos y su grupo familiar, pues prejuicios o información parcial pueden incrementar el estigma sobre el suicidio y, a la postre, disminuir la probabilidad de participación en estudios sobre la salud mental.

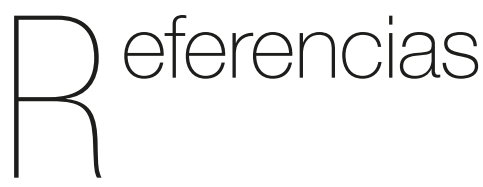

Álvarez, M., Colas, V., Barceló, M., Sánchez, Y., \& Fajardo, Y. (2017). Principales factores de riesgo relacionados con el intento suicida en un grupo de adolescentes. MEDISAN, 21(1), 157-163.

Bazán, J., Olórtegui, V., Vargas, H., \& Huayanay, L. (2016). Prevalencia y factores asociados con la conducta suicida en adolescentes de Lima rural. Revista de Neuropsiquiatría, 79(1), 3-15. DOI: https://doi.org/10.20453/ rnp.v79i1.2763.

Cárdenas, M. \& Arancibia, H. (2014). Potencia estadística y cálculo del tamaño del efecto en G*Power: Complementos a las pruebas de significación estadística y su aplicación en psicología. Salud \& Sociedad, 5(2), 210-224. DOI: 10.22199/ S07187475.2014.0002.00006.

Carvajal, G., \& Caro, C. (2011). Ideación suicida en la adolescencia: Una explicación desde tres de sus variables asociadas en Bogotá,
2009. Revista Colombia Médica, 42(2), 45-56.

Ceballos, G., Suarez, Y., Suescún, J., Gamarra, L., González, K., \& Sotelo, A. (2015). Ideación suicida, depresión y autoestima en adolescentes escolares de Santa Marta. Duazary, 12(1), 15-22. DOl: http:// dx.doi.org/10.21676/2389783X.1394.

Chamorro, E., \& Cristancho, D. (2010). Estudio epidemiológico de las conductas suicidas en estudiantes de secundaria de la ciudad de Ipiales. (Tesis de pregrado). Universidad de Nariño, Pasto. Recuperado de http://190.66.7.6:8085/atenea/ biblioteca/81946.pdf.

Cogollo, Z., Campo, A., \& Herazo, E. (2015). Escala de Rosenberg para autoestima: consistencia interna y dimensionalidad en estudiantes de Cartagena, Colombia. Psychologia. Avances de la Disciplina, 9(2), 61-71. DOI: http://dx.doi. org/10.21500/19002386.1814.

Congreso de Colombia (2012, 24 de abril). Ley 1523, por la cual se adopta la política nacional de gestión del riesgo de desastres y se establece el Sistema Nacional de Gestión del Riesgo de Desastres y se dictan otras disposiciones. Recuperado de http://repositorio.gestiondelriesgo.gov.co/ handle/20.500.11762/20575

Consejo Nacional de Política Económica y Social - CONPES. (2007). Lineamientos de política para implementar un proceso de gestión integral del riesgo en la zona de amenaza volcánica alta del volcán Galeras. Documento CONPES 3501. Bogotá. Recuperado de http://www.sena.edu.co/ downloads/2008/Planeacion/conpes\%20 3501\%20galeras.pdf. Consultado en septiembre 5 de 2010. 
Córdova, A., Estrada, A., \& Velázquez, M. (2013). Detección de riesgo suicida en una muestra de adolescentes estudiantes de escuelas secundarias. Revista Intercontinental de Psicología y Educación, 15(1), 7-21.

Cortés, A. (2013). Conducta suicida, adolescencia y riesgo. Revista Cubana de Medicina General Integral, 30(1), 132-13.

Cortés, A., Aguilar, J., Suárez, R., Rodríguez, E., \& Durán, J. (2011). Factores de riesgo asociados con el intento suicida y criterios sobre lo ocurrido en adolescentes. Revista Cubana de Medicina Integral, 27(1), 33-41.

Delgado, M. \& Santacruz, M. (2012). Caracterización psicométrica del Cuestionario de Eventos Estresantes Vitales CEVE-A-R en adolescentes de Pasto. (Trabajo de grado en Psicología). Pasto, Universidad de Nariño. Documento inédito. Recuperado de http://sired.udenar. edu.co/3987/1/86017.pdf.

Departamento Administrativo Nacional de Estadística -DANE. (2016). Defunciones no fetales por causa externa. Recuperado de https://www.dane.gov.co/files/ investigaciones/poblacion/2017/22diciembre-2017/nofetales2016/ Defunciones_causa_externa_2016def.xls.

Faul, F., Erdfelder, E., Lang, A., \& Buchner, A. (2007). G*Power 3: A flexible statistical power analysis program for the social, behavioral, and biomedical sciences. Behavior Research Methods, 39, 175-191. DOl: https://doi.org/10.3758/BF03193146.

Forero, I., Siabato, E., \& Salamanca, Y. (2017). Ideación suicida, funcionalidad familiar y consumo de alcohol en adolescentes de Colombia. Revista Latinoamericana de
Ciencias Sociales, Niñez y Juventud, 15(1), 431-442. DOI: http://dx.doi.org/10.11600/1 692715x.1512729042016

Gómez, Y., \& Villalobos, F. (2014). Competencias para la formulación de un proyecto de investigación. Guía metodológica para docentes investigadores. Pasto: Universidad de Nariño.

González, C., Chávez, A.M., Álvarez, M., Saldaña, A., Carreño, S., \& Pérez R. (2005). Prevalence of deliberate self-harm in adolescents students in Guanajuato State, México: Measurement: 2003. Social Behavior and Personality, 33(8), 777-792. DOl: https://doi.org/10.2224/ sbp.2005.33.8.777.

González, C.E. (2009). Propiedades psicométricas de la Escala de Desesperanza de Beck en una muestra bogotana. Psychología. Avances de la disciplina, 3(2), 17-30.

Hernández, A., González, I., \& López, A. (2013). Factores de riesgo relacionados con la conducta suicida en la infancia y adolescencia. MEDISAN, 17(12), 27-35.

IBM. (2013). IBM SPSS Statistics for Windows, Version 22.0. Armonk, NY: IBM Corp.

Jiménez, M., Hidalgo, J., Camargo, C. \& Dulce, B. (2014). El intento de suicidio en la población pediátrica, una alarmante realidad. Revista Ciencia de la Salud, 12(1), 59-83. DOl: http://dx.doi.org/10.12804/ revsalud12.1.2014.05.

Martínez, G. (2016). Descripción del intento de suicidio como constructo de violencia en la población pediátrica. Revista Facultad Nacional de Salud Pública, 34(1), 306-315. 
DOI: http://dx.doi.org/10.17533/udea.rfnsp. v34n3a05.

Martínez, J., \& Robles, A. (2016). Percepción de actores sociales sobre la conducta suicida: Análisis de contenido a través de grupos focales. Informes Psicológicos, 16(2), 53-68. DOl: http://dx.doi.org/10.18566/ infpsicv16n2a04

Matud, M. P. (1998). Investigación del estrés y su impacto en la salud de las mujeres en Canarias. Informe del proyecto de investigación. Instituto Canario de la Mujer.

Matud, P., Carballeira, M., López, M., Marrero, R., \& Ibáñez, I. (2002). Apoyo social y salud: Un análisis de género. Salud Mental, 25, 32-37.

Miller, A., Esposito-Smythers, C. \& Leichtweis, R. (2015). Role of social support in adolescent suicidal ideation and suicide attempts. Journal of Adolescent Health, 56(3), 286-292. DOI: 10.1016/j. jadohealth.2014.10.265.

Ministerio de Salud (2015). Encuesta Nacional de Salud Mental 2015. Bogotá, Colombia: Ministerio de Salud. Recuperado de http:// www.visiondiweb.com/insight/lecturas/ Encuesta_Nacional_de_Salud_Mental_ Tomo_I.pdf

Ministerio de Salud de Colombia (1993). Resolución 8430, por la cual se establecen las normas científicas, técnicas y administrativas para la investigación en salud. Recuperado de https://www.minsalud.gov.co/sites/rid/ Lists/BibliotecaDigital/RIDE/DE/DIJ/ RESOLUCION-8430-DE-1993.PDF

Ministerio de Salud y Protección Social. (2017). Boletín de salud mental: Conducta suicida.
Boletín del Observatorio Nacional de Salud Mental, 2, 1-19. Recuperado de https:// www.minsalud.gov.co/sites/rid/Lists/ BibliotecaDigital/RIDE/VS/PP/ENT/boletinconducta-suicida.pdf.

Miranda, I., Cubillas, M., Pérez, R., \& Valdez, E. (2009). Ideación suicida en población escolarizada infantil: factores psicológicos asociados. Salud Mental, 32(6), 495-502.

Montoya, B. (2018). Comportamiento del Suicidio. Colombia, 2017. Forensis. Datos para la vida, 19(1), 349-385.

Ojeda, E. (2008). Problemática psicosocial en comunidades residentes en la Zona de Amenaza Volcánica Alta del Volcán Galeras (ZAVA): Una perspectiva desde la Psicología Comunitaria para el acompañamiento psicosocial. Universidad y Salud, 9(1), 45-64.

Organización Mundial de la Salud - OMS (2000). Prevención del suicidio: un instrumento para médicos generalistas. Recuperado de http://www.who.int/mental_health/media/ general_physicians_spanish.pdf.

Organización Mundial de la Salud. (2018, enero). Suicidio. Recuperado de http:// www.who.int/mediacentre/factsheets/ fs398/es/

Pérez, M., Martínez, L., Vianchá, M., \& Avendaño, B. (2017). Intento e ideación suicida y su asociación con el abuso sexual en adolescentes escolarizados de Boyacá-Colombia. Revista Diversitas: Perspectivas en Psicología, 13(1), 91101. DOl: http://dx.doi.org/10.15332/ s1794-9998.2017.0001.07.

Pérez, l., Téllez, D., Vélez, A., \& Ibáñez, M. (2012). Caracterización de factores asociados con 
comportamiento suicida en adolescentes estudiantes de octavo grado, en tres colegios bogotanos. Revista Colombiana de Psiquiatría, 41(1), 26-47. DOI: 10.1016/ S0034-7450(14)60067-6.

Pérez-Chan, M. (2015). Familia multiproblemática como factor en el desarrollo de la ideación e intento suicida en adolescentes. Salud en Tabasco, 21(23), $45-54$.

Piedrahita, L., García, M., Mesa, J., \& Rosero, E. (2011). Identificación de los factores relacionados con el intento de suicidio, en niños y adolescentes a partir de la aplicación del proceso de atención de enfermería. Colombia Médica, 42(3), 334-341.

Piedrahita, L., Paz, K., \& Romero, A. (2012). Estrategia de intervención para la prevención del suicidio en adolescentes: La escuela como contexto. Revista hacia la Promoción de la Salud, 17(2), 136-148.

Quiceno, J., \& Vinaccia, S. (2013). Calidad de vida, factores salutogénicos e ideación suicida en adolescentes. Terapia Psicológica, 31(1), 263271. DOl: http://dx.doi.org/10.4067/ S0718-48082013000200012.

Radloff, L.S. (1977). The CES-D Scale: A self report depression scale for research in the general population. Journal of Applied Psychological Measurement, 1, 385-401. DOl: https://doi. org/10.1177/014662167700100306.

República de Colombia. (2005, 15 de noviembre). Decreto 4106, por el cual se declara la existencia de una situación de desastre en los municipios de Pasto, Nariño y la Florida, en el Departamento de Nariño. Recuperado de http://www. suin-juriscol.gov.co/clp/contenidos.dll/ Decretos/1893038?fn=document-frame. $\mathrm{htm} \$ \mathrm{f}=$ templates $\$ 3.0$

República de Colombia. (2008, 7 de octubre). Decreto 3905, por el cual, en desarrollo del Decreto-ley 919 de 1989, se definen el objeto y los instrumentos necesarios para la implementación del Plan de Reasentamiento en la Zona de Amenaza Volcánica Alta (ZAVA) del Volcán Galeras, declarada como zona de desastre por el Decreto 4106 de 2005. Recuperado de http://www.minambiente. gov.co/images/normativa/decretos/2008/ dec_3905_2008.pdf

Salas, M., Levette, M., Redondo, J., \& Luzardo, M. (2017). Ideación suicida en adolescentes víctimas de cyberbullyng del Instituto Empresarial Gabriela Mistral de Floridablanca (Santander). Revista Virtual Universidad Católica del Norte, 51, 80-97.

Sánchez, D., García, A., \& Muela, J. (2014). Preventions, assessment and treatment of suicidal behavior. Anales de Psicología, 30(3), 952-963. DOl: https://doi. org/10.6018/analesps.30.3.148691.

Sánchez, R., Guzmán, Y., \& Cáceres, H. (2005). Estudio de la imitación como factor de riesgo para ideación suicida en estudiantes universitarios adolescentes. Revista Colombiana de Psiquiatría, 34(1), 12-25.

Sánchez, L., Morfín, T., García, J., Quintanilla, R., Hernández, R., Contreras, E., \& Cruz, J. (2014). Intento de suicidio en Adolescentes Mexicanos: Perspectiva desde el Consenso Cultural. Acta de Investigación Psicológica, 4(1), 1446-1458. DOl: https:// doi.org/10.1016/S2007-4719(14)70386-2. 
Siabato, E. \& Salamanca, Y. (2015). Factores asociados a ideación suicida en universitarios. Psychologia: Avances de la Disciplina, 9(1), 71-81. DOl: http://dx.doi. org/10.21500/19002386.994.

Toro, D., Paniagua, R., González, C., \& Montoya, B. (2009). Caracterización de adolescentes escolarizados con riesgo de suicidio, Medellín, 2006. Facultad Nacional de Salud Pública, 27(3), 302-308.

Unikel, C., Gómez, G., \& González-Forteza, C. (2006). Suicidal Behaviour and Psychosocial Correlates in Mexican women with eating disorders. European Eating Disorders Review, 14(6), 414-421. DOl: https://doi.org/10.1002/erv.707.

Valdivia, M., Silva, D., Sanhueza, F., Cova, F., \& Melipillán, R. (2015). Prevalencia de intento de suicidio en adolescentes y factores de riesgo asociados en una comuna rural de la provincia de Concepción. Revista de Medicina de Chile, 143, 320328. DOl: http://dx.doi.org/10.4067/ S0034-98872015000300006.

Vargas, H., \& Saavedra, J. (2012). Factores asociados con la conducta suicida en adolescentes. Revista de Neuropsiquiatría, 75(1), 19-28. DOl: https://doi.org/10.20453/ rnp.v75i1.1539.
Vásquez, R., \& Quijano, M. (2013). Cuando el intento de suicidio es cosa de niños. Revista Colombiana de Psiquiatría, 43(1), 36-46. DOl: http://dx.doi.org/10.1016/j. rcp.2013.11.004.

Veytia, M., Fajardo, R., Guadarrama, R., \& Escutia, N. (2016). Inteligencia Emocional: factor positivo ante la depresión en adolescentes de bachillerato. Informes Psicológicos, 16(1), 35-50 http://dx.doi. org/10.18566/infpsicv16n1a02

Vianchá, M., Bahamon, M., \& Alarcón, L. (2013). Variables psicosociales asociadas al intento suicida, ideación suicida y suicidio en jóvenes. Tesis Psicológica, 8(1), 112-123.

Villalobos, F. (2008). Aplicación del modelo procesual del estrés a las conductas suicidas. Universidady Salud, 1(8), 125-140.

Villalobos, F. (2009). Situación de la conducta suicida en estudiantes de colegios y universidades de San Juan de Pasto, Colombia. Salud Mental, 31, 165-171.

Villalobos, F., \& Ortiz, L. (2012). Características psicométricas de la escala CES-D en adolescentes de San Juan de Pasto. Avances en Psicología Latinoamericana, 30(2), 328-340. 